\title{
The Relation between the Quasi-Localized Energy-Momentum Complexes and the Thermodynamic Potential for the Schwarzschild-de Sitter Black Hole
}

\author{
I-Ching Yang \\ Department of Applied Science, National Taitung University, Taitung 95002, Taiwan \\ Correspondence should be addressed to I-Ching Yang; icyang@nttu.edu.tw
}

Received 25 August 2017; Revised 21 October 2017; Accepted 31 October 2017; Published 14 December 2017

Academic Editor: Elias C. Vagenas

Copyright (C) 2017 I-Ching Yang. This is an open access article distributed under the Creative Commons Attribution License, which permits unrestricted use, distribution, and reproduction in any medium, provided the original work is properly cited. The publication of this article was funded by $\mathrm{SCOAP}^{3}$.

The Schwarzschild-de Sitter black hole solution, which has two event horizons, is considered to examine the relation between the energy component of quasi-localized energy-momentum complexes on $\mathscr{M}$ and the heat flows passing through its boundary $\partial \mathscr{M}$. Here $\mathscr{M}$ is the patch between cosmological event horizon and black hole event horizon of the SdS black hole solution. Conclusively, the relation, like the Legendre transformation, between the energy component of quasi-localized Einstein and Møller energymomentum complex and the heat flows passing through the boundary is obeyed, and these two energy components of quasilocalized energy-momentum complexes could be corresponding to thermodynamic potentials.

\section{Introduction}

Recently, Yang et al. [1-3] have inferred that the formula about the quasi-localized Einstein and Møller energy-momentum complexes on $\mathscr{M}^{*}$ and the heat flows passing through the boundary of $\mathscr{M}^{*}$ are related as

$$
\left.E_{\mathrm{E}}\right|_{\mathscr{M}^{*}}=\left.E_{\mathrm{M}}\right|_{\mathscr{M}^{*}}-\sum_{\partial \mathscr{M}^{*}} \mathrm{TS}
$$

Here $\mathscr{M}^{*}$ is the patch between event horizon $\mathscr{H}_{+}$located at $r=r_{+}$and inner Cauchy horizon $\mathscr{H}_{-}$located at $r=r_{-}$ for the spherically symmetric black hole with two separate horizons. Equation (1) is similar to the Legendre transformation between Helmholtz free energy $F$ and internal energy $U$ or between Gibbs free energy $G$ and enthalpy $H$, and therefore these energy components of quasi-localized energymomentum complexes $\left.E_{\mathrm{E}}\right|_{\mathscr{M}^{*}}$ and $\left.E_{\mathrm{M}}\right|_{\mathscr{M}^{*}}$ could correspond to thermodynamic potentials. But, in previous studies of Yang et al. [1-3], $r_{-}$is a Cauchy horizon. There was a controversy that an acceptable definition exists for the temperature and entropy of black hole at Cauchy horizon. In this article, I consider the Schwarzschild-de Sitter (SdS) black hole solution, which has two separate event horizons, and a review of relation between the energy component of quasi-localized energy-momentum complexes on a patch between two event horizons and the heat flows passing through its boundary.

\section{The Schwarzschild-de Sitter Black Hole Metric}

The SdS black hole solution [4], describing a spherically symmetric solution of the vaccum Einstein field equations in the presence of a positive cosmological constant $\Lambda$

$$
R_{\mu \nu}-\frac{1}{2} g_{\mu \nu} R+\Lambda g_{\mu \nu}=0
$$

is given in the static form

$$
d s^{2}=f(r) d t^{2}-f^{-1}(r) d r^{2}-r^{2} d \Omega
$$

and its metric function was found as

$$
f(r)=1-\frac{2 M}{r}-\frac{r^{2}}{b^{2}},
$$




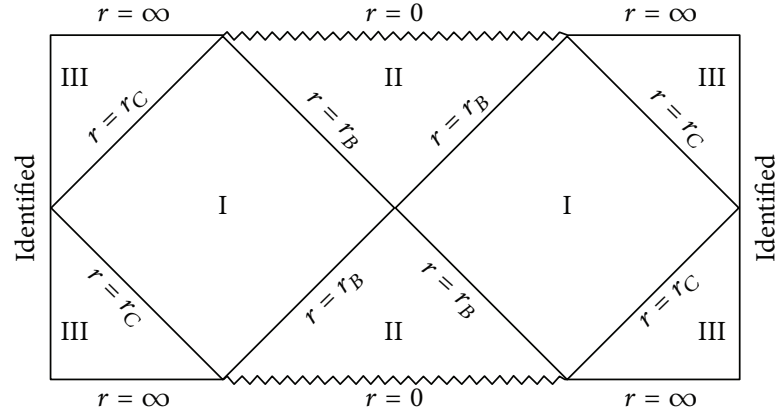

Figure 1: Penrose diagram for SdS black hole solution.

where $b^{2}=3 / \Lambda$. Here, I shall consider the metric function in a factorization form

$$
f(r)=-\frac{1}{r b^{2}}\left(r-r_{c}\right)\left(r-r_{b}\right)\left(r-r_{0}\right) .
$$

When $3 \sqrt{3} M / b<1$, the metric function has two distinct positive real roots $r_{c}$ and $r_{b}$, and the smaller one $r_{b}$ and the larger one $r_{c}$ can be regarded as the position of the black hole event horizon and the cosmological event horizon for observers moving on the world lines of constant $r$ between $r_{b}$ and $r_{c}$. Compared with (4), the relations for these three roots are given by

$$
\begin{aligned}
r_{c}+r_{b}+r_{0} & =0, \\
r_{c} r_{b}+r_{c} r_{0}+r_{b} r_{0} & =-b^{2}, \\
r_{c} r_{b} r_{0} & =-2 M b^{2} .
\end{aligned}
$$

Because of (6), $r_{0}=-\left(r_{c}+r_{b}\right)$, the metric function is taken to be

$$
f(r)=-\frac{1}{r b^{2}}\left(r-r_{c}\right)\left(r-r_{b}\right)\left(r+r_{c}+r_{b}\right),
$$

and (7) and (8) are also reorganized as

$$
\begin{aligned}
& r_{c}^{2}+r_{c} r_{b}+r_{b}^{2}=b^{2}, \\
& r_{c} r_{b}\left(r_{c}+r_{b}\right)=2 M b^{2} .
\end{aligned}
$$

Let $\mathcal{S}^{2}(r)$ be a 2 -sphere of radius $r$. Thus we suggest that $\mathscr{M}=$ $\left\{\mathcal{S}^{2}(r) \mid r_{c}>r>r_{b}\right\}$ is the patch between cosmological event horizon $\mathscr{H}_{C}=\mathcal{S}^{2}\left(r_{c}\right)$ and black hole event horizon $\mathscr{H}_{B}=$ $\mathcal{S}^{2}\left(r_{b}\right)$, and the boundary of $\mathscr{M}$ is $\partial \mathscr{M}=\mathscr{H}_{C} \cup \mathscr{H}_{B}$. The patch $\mathscr{M}$ is region I of Penrose diagram for SdS black hole solution (shown as Figure 1).

\section{The Thermodynamics of SdS Black Hole}

In the study of the thermodynamics of SdS black hole by Gibbons and Hawking [5], the Hawking temperatures T [6] of $\mathscr{H}_{C}$ and $\mathscr{H}_{B}$ are

$$
\begin{aligned}
\left.\mathbf{T}\right|_{\mathscr{H}_{\mathscr{C}}} & =\frac{1}{4 \pi r_{c} b^{2}}\left(r_{c}-r_{b}\right)\left(2 r_{c}+r_{b}\right), \\
\left.\mathbf{T}\right|_{\mathscr{H}_{\mathscr{B}}} & =\frac{1}{4 \pi r_{b} b^{2}}\left(r_{c}-r_{b}\right)\left(r_{c}+2 r_{b}\right),
\end{aligned}
$$

and the Bekenstein-Hawking entropies $S[7,8]$ of $\mathscr{H}_{C}$ and $\mathscr{H}_{B}$ are

$$
\begin{aligned}
\left.\mathrm{S}\right|_{\mathscr{H}_{\mathscr{C}}} & =\pi r_{c}^{2}, \\
\left.\mathbf{S}\right|_{\mathscr{H}_{\mathscr{B}}} & =\pi r_{b}^{2} .
\end{aligned}
$$

For those two event horizons, the heat flows are evaluated as

$$
\begin{aligned}
\left.\mathbf{T S}\right|_{\mathscr{H}_{\mathscr{C}}} & =\frac{1}{4 b^{2}}\left(r_{c}-r_{b}\right)\left(2 r_{c}^{2}+r_{c} r_{b}\right), \\
\mathbf{T S} & \left.\right|_{\mathscr{H}_{\mathscr{B}}}=\frac{1}{4 b^{2}}\left(r_{c}-r_{b}\right)\left(r_{c} r_{b}+2 r_{b}^{2}\right) .
\end{aligned}
$$

Hence, the heat flow passing through the boundary $\partial \mathscr{M}$ would be expressed by

$$
\left.\mathbf{T S}\right|_{\partial \mathscr{M}}=\frac{1}{2 b^{2}}\left(r_{c}^{3}-r_{b}^{3}\right)
$$

\section{The Quasi-Localized Energy-Momentum Complexes}

Subsequently, on $\mathscr{M}$, the quasi-localized energy-momentum complexes in the Trautman [9], Einstein [10] and Møller [11, 12] prescription should be considered. The energy component of the Einstein energy-momentum complex $[9,10]$ is given by

$$
E_{\mathrm{E}}=\frac{1}{16 \pi} \oint H_{0}{ }^{0 i} \widehat{n}_{i} \cdot \overrightarrow{d S}
$$

where

$$
H_{0}{ }^{0 i}=\frac{g_{00}}{\sqrt{-g}} \frac{\partial}{\partial x^{m}}\left[(-g) g^{00} g^{i m}\right]
$$

and $\widehat{n}_{i}$ is the outward unit normal vector over the infinitesimal surface element $\overrightarrow{d S}$. The energy component within radius $r$ obtained by the Einstein energy-momentum complex is

$$
E_{\mathrm{E}}=\frac{r}{2}(1-f)
$$

Therefore, the energy component of quasi-localized Einstein energy-momentum complex on $\mathscr{M}$ is

$$
\left.E_{\mathrm{E}}\right|_{r_{b}} ^{r_{c}}=\frac{1}{2}\left(r_{c}-r_{b}\right)=\frac{1}{2 b^{2}}\left(r_{c}^{3}-r_{b}^{3}\right)
$$

Moreover, according to the definition of the Møller energymomentum complex [11,12] and Gauss's theorem, its energy component is given as

$$
E_{\mathrm{M}}=\frac{1}{8 \pi} \oint \chi_{0}{ }^{0 i} \widehat{n}_{i} \cdot \overrightarrow{d S}
$$

where

$$
\chi_{0}^{0 i}=\sqrt{-g}\left(-\frac{\partial g_{00}}{\partial x^{i}}\right) g^{00} g^{i i}
$$


So the energy component with radius $r$ obtained using the Møller energy-momentum complex is

$$
\begin{aligned}
E_{\mathrm{M}} & =\frac{r^{2}}{2} \frac{d f}{d r}=-\frac{r}{2} f-\frac{r}{2 b^{2}}\left[\left(r-r_{c}\right)\left(r+r_{b}+r_{c}\right)\right. \\
& \left.+\left(r-r_{b}\right)\left(r+r_{b}+r_{c}\right)+\left(r-r_{b}\right)\left(r-r_{c}\right)\right],
\end{aligned}
$$

and the energy component of quasi-localized Møller energymomentum complex on $\mathscr{M}$ is

$$
\begin{aligned}
& \left.E_{\mathrm{M}}\right|_{r_{b}} ^{r_{c}}=-\frac{1}{2 b^{2}}\left[r_{c}\left(r_{c}-r_{b}\right)\left(2 r_{c}+r_{b}\right)\right. \\
& \left.\quad-r_{b}\left(r_{b}-r_{c}\right)\left(r_{c}+2 r_{b}\right)\right]=-\frac{1}{b^{2}}\left(r_{c}^{3}-r_{b}^{3}\right) .
\end{aligned}
$$

\section{Conclusion}

Consequently, the difference of energy between the Einstein and Møller prescription [13] is defined as

$$
\Delta E=E_{\mathrm{E}}-E_{\mathrm{M}}
$$

According to (18) and (22), the difference of energy in the patch $\mathscr{M}$ is

$$
\left.\Delta E\right|_{\mathscr{M}}=\frac{3}{2 b^{2}}\left(r_{c}^{3}-r_{b}^{3}\right),
$$

and its value is three times the heat flow passing through the boundary $\partial \mathscr{M}$

$$
\left.\Delta E\right|_{\mathscr{M}}=\left.3 \mathrm{TS}\right|_{\partial \mathscr{M}}
$$

In this way, the energy components of quasi-localized Einstein energy-momentum complex $\left.E_{\mathrm{E}}\right|_{\mathcal{M}}$ and Møller energymomentum complex $\left.E_{\mathrm{M}}\right|_{\mathscr{M}}$ will combine with the heat flow passing through the boundary $\partial \mathscr{M}$

$$
\left.E_{\mathrm{E}}\right|_{\mathscr{M}}=\left.E_{\mathrm{M}}\right|_{\mathscr{M}}+\left.3 \mathrm{TS}\right|_{\partial \mathscr{M}}
$$

although the factor " +3 " of the heat flow passing through $\partial \mathscr{M}$ in (26) is different from the factor " -1 " in (1). As a matter of fact, $\left.E_{\mathrm{M}}\right|_{\mathscr{M}}$ must be positive if $\mathscr{M}$ is dominated by attractive gravitation. For that reason, I prefer that $E_{\mathrm{M}} \mathrm{I}_{\mathscr{M}}$ is replaced by its absolute value $\left|E_{\mathrm{M}}\right|_{\mathscr{M}} \mid$. Finally, the difference of energy between the Einstein and Møller prescription on the patch $\mathscr{M}$ is equal to the heat flow passing through its boundary $\partial \mathscr{M}$, as the formula previously pointed out [1]

$$
\left.E_{\mathrm{E}}\right|_{\mathscr{M}}=\left|E_{\mathrm{M}}\right|_{\mathscr{M}} \mid-\sum_{\partial \mathscr{M}} \mathrm{TS}
$$

Because all boundaries of $\mathscr{M}$ are event horizons, the summation of the heat flows passing through those boundaries $\sum_{\partial \mathscr{M}}$ TS is well defined. In conclusion, for the SdS black hole solution, the establishment of Legendre transformation in (27) exhibits that $\left.E_{\mathrm{E}}\right|_{\mathscr{M}}$ and $\left|E_{\mathrm{M}}\right|_{\mathscr{M}} \mid$ would play the role of thermodynamic potential. This result conforms with the viewpoint of Chang et al. [14] and our latest studies [1-3].

\section{Conflicts of Interest}

There are no conflicts of interest related to this paper.

\section{Acknowledgments}

The author would like to thank Professor Ching-Tang Tsao for useful suggestions. This work was partially supported by the Ministry of Science and Technology (Taiwan) under Contract no. MOST103-2633-M143-001.

\section{References}

[1] I.-C. Yang, "The quasi-localized Einstein and Møller energy complex as thermodynamic potentials," Chinese Journal of Physics, 50, 544, 2012.

[2] I.-C. Yang, B.-A. Chen, and C.-C. Tsai, “Thermodynamical properties and quasi-localized energy of the stringy dyonic black hole solution," Modern Physics Letters, A27, 1250169, 2012.

[3] I.-C. Yang and W.-K. Huang, "The thermodynamical properties of charged dilaton-axion black hole and its quasi-localized energy," Modern Physics Letters A, vol. 29, no. 23, Article ID 1450126, 2014.

[4] F. Kottler, "Über die physikalischen Grundlagen der Einsteinschen Gravitationstheorie," Annals of Physics, 261, 401, 1918.

[5] G. W. Gibbons and S. W. Hawking, "Cosmological event horizons, thermodynamics, and particle creation," Physical Review D: Covering Particles, Fields, Gravitation, and Cosmology, vol. 15, no. 10, 2738 pages, 1977.

[6] M. Visser, "Dirty black holes: thermodynamics and horizon structure," Physical Review D: Covering Particles, Fields, Gravitation, and Cosmology, vol. 46, no. 6, pp. 2445-2451, 1992.

[7] S. W. Hawking, "Black hole explosions?" Nature, vol. 248, no. 5443, pp. 30-31, 1974.

[8] S. W. Hawking, "Particle creation by black holes," Communications in Mathematical Physics, vol. 43, no. 3, pp. 199-220, 1975.

[9] A. Trautman, Gravitation: An Introduction to Current Research, L. Witten, Ed., Witten, New York, NY, USA, 1962.

[10] A. Einstein, "Zür allgemeinen Relativitätstheorie," Preuss. Akad. Wiss. Berlin, 47, 778-799, 1915.

[11] C. Møller, "On the localization of the energy of a physical system in the general theory of relativity," Annals of Physics, vol. 4, no. 4, pp. 347-371, 1958.

[12] C. Møller, "Further remarks on the localization of the energy in the general theory of relativity," Annals of Physics, vol. 12, no. 1, pp. 118-133, 1961.

[13] I.-C. Yang and I. Radinschi, "On the difference of energy between the Einstein and Møller prescription," Chinese Journal of Physics, vol. 42, no. 1, pp. 40-44, 2004.

[14] C.-C. Chang, J. M. Nester, and C.-M. Chen, "Pseudotensors and quasilocal energy-momentum," Physical Review Letters, vol. 83, no. 10, pp. 1897-1901, 1999. 

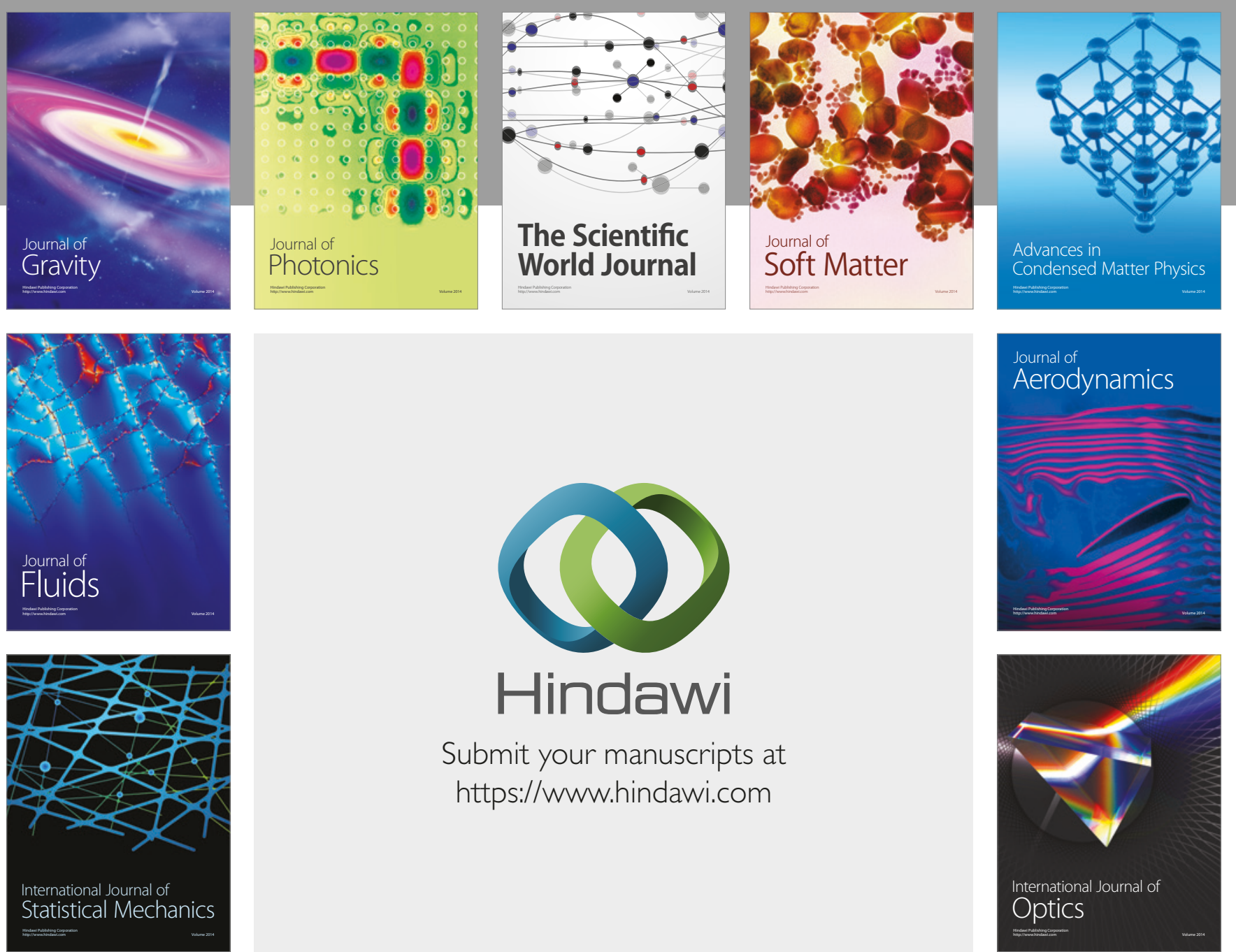

Submit your manuscripts at

https://www.hindawi.com
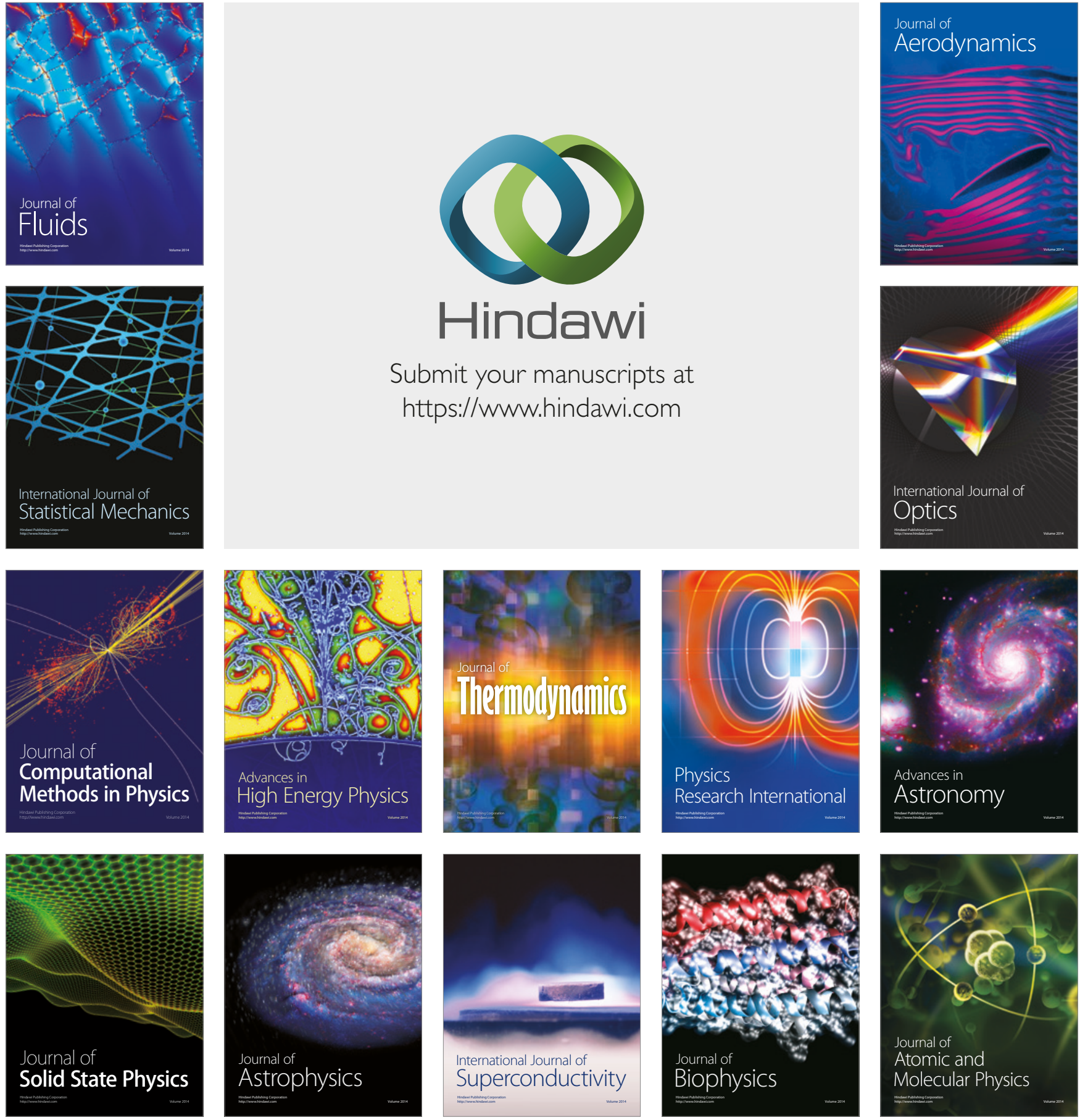